\title{
Assessment of impact of varied preconditioning factors, used in isolation and combination, for enhancement of cytokine secretions in stem cell conditioned medium
}

\author{
Rajashri Mokashi*, Debjani Dasgupta \\ School of Biotechnology and Bioinformatics, D Y Patil University, Navi Mumbai, India
}

\begin{tabular}{l}
\hline ARTICLE INFO \\
\hline Article history: \\
Received on: August 31, 2019 \\
Accepted on: March 08, 2020 \\
Available online: May 26, 2020 \\
\hline
\end{tabular}

Key words:

Dental pulp stem cell, conditioned medium, preconditioning factors, cytokines, VEGF, HGF

\begin{abstract}
Stem cell conditioned medium exhibits a huge regenerative potential but low concentration of cytokines, high development cost, and scalability challenges are major deterrents to product advances. This research studied impact on vascular endothelial growth factor (VEGF) and hepatocyte growth factor (HGF) secretions after preconditioning human dental pulp stem cells (HDPSCs) with diverse factors, viz., Deferoxamine $(250 \mu \mathrm{M})$, Epidermal Growth Factor (EGF) (2 ng/ml), Angiotensin I (5mM) and Insulin Transferrin Selenium (ITS) (1\%), and Niacinamide $(5 \mathrm{mM})$. Additionally, advantage of using "pooled population" of HDPSCs was ascertained. HDPSCs were incubated under standard culture conditions using optimized growth media. On reaching $80 \%-$ $85 \%$ confluency, media was discarded and fresh serum free media with preconditioning factors, initially one at a time and subsequently in combination, was added. Spent media, collected after 48 hours of incubation, was used for enzyme-linked immunosorbent assay testing of selected cytokines. In comparison to control, preconditioning factors, used in isolation showed varied but significant upregulation in the secretion of VEGF but down regulation of HGF. When used in combination, VEGF secretion increased almost 10 times of control with no significant change in HGF level. Results demonstrated importance of choosing right preconditioning factors and right interplay of preconditioning factors to enhance secretions. This research paves the path to develop an effective and commercially viable conditioned medium that can be used as "biological active" for pharmaceuticals and for developing "customized" serum free medium enriched with selected cytokines.
\end{abstract}

\section{INTRODUCTION}

Scientists see a huge potential in using conditioned medium as "biological active" to treat a number of ailments wherein paracrine effect creates desired results for stem cells $[1,2]$. Studies reveal that level and type of paracrine factors secreted by different stem cell resources play an important role in influencing cell recruitment and tissue repair. Although scientists have been successful in deriving these trophic factors in the laboratory, low concentrations of growth factors/cytokines, and high cost for developing stem cell conditioned medium are major deterrents to product advances. Recent studies show that Mesenchymal

\footnotetext{
*Corresponding Author

Rajashri Mokashi, School of Biotechnology and Bioinformatics, D Y Patil University, Mumbai, India.

E-mail: rajashri.mokashi@gmail.com
}

Stem Cells (MSCs) can modulate pattern, quantity, and type of secretions in vitro through a preconditioning process [3-5]. This is a major advancement as it opens up new avenues of creating conditioned medium of choice through an interplay of preconditioning factors and gives rise to the possibility of influencing these parameters in order to increase the output of any particular growth factor/cytokine. However, there are no reports studying the relative impact of different preconditioning factors on secretions when used individually and synergistic impact when used in combination. The emphasis of this research was to develop "commercially viable" biological active along with "efficacy". It was postulated that source of stem cell will play a pivotal role in preparation of conditioned medium and a robust "Master Bank" and "Working Cell Bank," which can be utilized over multiple outputs to ensure reproducibility and scalability, will be imperative. Human Dental pulp was identified as the stem cell source owing to ease and convenience in collection, 
an important factor to build a large Master Bank [6]. Better proliferation rates and differentiation capacity in comparison to bone marrow, cord tissue, or adipose tissue reinforced the choice of dental pulp stem cells for developing the conditioned medium [7]. This research work was based on following hypotheses- (1) Mesenchymal stem cells from Dental pulp would have significant advantage in quantity over mesenchymal cells isolated from other sources. (2) Pooled population of mesenchymal stem cells would offer better cell yield and viability for developing conditioned medium. (3) Presence of preconditioning factors would influence secretion of growth factors from pooled dental pulp stem cells (DPSC) population when used in isolation or in combination. This research, hence, focused on validation of dental pulp stem cell advantage over other sources and evaluation of hypothesized benefit of using 'pooled' stem cell population. An exploratory route was taken to identify various preconditioning factors that are likely to influence output of secretions. Through this research, relative impact of using preconditioning factors in isolation and combination was also studied. This study may help in the development of conditioned medium product, which is effective, scalable, reproducible, commercially viable, and customized to enhance specific secretions.

\section{MATERIALS AND METHODS}

\subsection{Preparation of Master Cell Bank}

Dental tissue was harvested in an aseptic environment. Chopped pieces of dental pulp tissue were allowed to adhere to cell culture dish in presence of growth media - Alpha Minimum Essential Medium- Alpha MEM with 20\% fetal bovine serum (FBS) $+2 \mathrm{mM}$ Glutamine $+100 \mathrm{mM}$ ascorbic Acid + Penicillin/Streptomycin $(100 \mu \mathrm{g} / \mathrm{ml})$. Within a short time, cells migrated out of the tissue over the surface of the culture plate. Primary cells, thus obtained, were sub-cultured over multiple passages after transferring to fresh culture plates and finally cryopreserved in DMSO.

\subsection{Comparison with Stem Cells from Other Sources}

Cell cultures were initiated with 1,000 cells $/ \mathrm{cm}^{2}$ in 6 -well plates with MSCs from different stem cell sources, such as dental pulp, adipose tissue, umbilical cord, and bone marrow. Cells were allowed to reach confluence in Alpha MEM supplemented with $20 \%$ FBS, $2 \mathrm{mM}$ Glutamine, 100mM ascorbic Acid and Penicillin/Streptomycin $(100 \mu \mathrm{g} / \mathrm{ml})$ as normal culture conditions. Cells were washed with Dulbecco's Phosphate Buffered Saline (DPBS) and detached using $0.25 \%$ trypsin. Cells were then counted

\subsection{Preparation of Working Cell Bank}

Five different samples of dental pulp stem cells maintained in Master Bank were thawed and equal concentration of cells that needed to be pooled were taken. Samples were seeded in T25 flask at a density of 5,000 cells $/ \mathrm{cm}^{2}$. Therefore, 1,25,000 cells were seeded in a T25 flask (approximately 25,000 cells were taken from each sample). Pooled suspension was inoculated in a sterile T25 flask and incubated in $\mathrm{CO}_{2}$ incubator at normal culture conditions. Cells were incubated till $80 \%-90 \%$ confluency was attained. These cells were then cryopreserved in Dimethyl Sulfoxide (DMSO) for future use.

\subsection{Production of Standard Conditioned Medium}

\subsubsection{Preparation of growth media for MSCs}

Growth medium was prepared as follows -

Alpha MEM with 20\% FBS + 2mM Glutamine $+100 \mathrm{mM}$ ascorbic Acid + Penicillin/Streptomycin $(100 \mu \mathrm{g} / \mathrm{ml})$

\subsubsection{Revival of cryostock for production of conditioned medium using serum free media}

One milliliter of cryopreserved cell stock at passage level 5 (P5) was thawed and transferred in a $15 \mathrm{ml}$ centrifuge tube. $2 \mathrm{ml}$ of media was added slowly to $15 \mathrm{ml}$ centrifuge tube. Tube was centrifuged for 5 minutes at 1,500 rpm and supernatant was discarded. Cell pellet was re-suspended in $1 \mathrm{ml}$ PBS. Tube was centrifuged for 5 minutes at 1,500 rpm and supernatant was discarded. Cell pellet was re-suspended in $1 \mathrm{ml}$ of media and cells were seeded in appropriate flask/plate and $4 \mathrm{ml}$ of media was added. Flask was incubated in $\mathrm{CO}_{2}$ incubator at normal culture conditions. At $80 \%-85 \%$ confluency, spent media was collected and discarded and cells were washed after addition of $1 \mathrm{ml}$ of PBS to appropriate flask/plate. Fresh serum free media was added. Flask/plate was incubated in $\mathrm{CO}_{2}$ incubator at $37^{\circ} \mathrm{C}$, $20 \%$ oxygen and $\mathrm{pH} 7.4$ for 48 hours. After 48 hours, spent media was collected and used for enzyme-linked immunosorbent assay (ELISA) testing of selected cytokine. Analysis was conducted for vascular endothelial growth factor (VEGF) and hepatocyte growth factor (HGF). All experiments were conducted in triplicates and the average readings were used for analysis.

\subsubsection{Cytokine testing of conditioned medium}

Testing was conducted as per the user manuals of the ELISA kits (Invitrogen) for the selected cytokines. Viz. VEGF and HGF.

\subsection{Testing of Cytokine Secretion using Different Preconditioning Factors}

Cells were revived at passage 5 level (P5) in T25 flask and incubated in $\mathrm{CO}_{2}$ incubator at $37^{\circ} \mathrm{C}, 20 \%$ oxygen and $\mathrm{pH} 7.4$ for 48 hours. Media was discarded after cells reached $80-85 \%$ confluency $1 \mathrm{ml}$ PBS was added and appropriate flask/plate was washed. Fresh serum free media was added with preconditioning factors (one at a time and subsequently all together). The preconditioning factors tested were as follows: EGF-2 ng/ml, Niacinamide $-5 \mathrm{mM}$, insulin transferrin selenium (ITS) - $1 \%$, Angiotensin-100 nM, Deferoxamine - $250 \mu \mathrm{M}$. Appropriate flask/plate was incubated in $\mathrm{CO}_{2}$ incubator at $37^{\circ} \mathrm{C}$ for 48 hours. Spent media was collected after 48 hours and used for ELISA testing.

\section{RESULTS AND DISCUSSION}

\subsection{Comparison of Growth Kinetics of Dental Pulp Stem Cells with Stem Cells from Other Sources}

Objective of this study was to confirm dental pulp stem cell advantage over others through comparison of growth kinetics of mesenchymal stem cells obtained from dental pulp and other sources viz. umbilical cord, bone marrow and adipose tissue. 
While mesenchymal stem cells are present in multiple tissues of the body, they differ in population numbers and proliferation capacities as per anatomical location. Pittenger et al have reported 500 times more cells from adipose tissue when compared with equivalent amount of bone marrow mesenchymal stem cells. Studies have demonstrated higher proliferation rates of umbilical cord MSCs and adipose tissue derived MSCs in comparison to bone marrow. Comparative study performed by Lu et al revealed significantly slower population doubling (PD) times for BM MSCs against UC - MSCs. Peng et al listed PD times of 24 hours for umbilical cord MSCs, 45.2 hours for adipose derived MSCs and 61.2 hours for bone marrow mesenchymal stem cells [8].

In this study, growth kinetics of mesenchymal stem cells isolated from various sources were studied. For drawing conclusions, it would have been ideal to compare stem cells from various sources isolated from a similar age group, if not same individual but it was practically impossible. Yet, an attempt was made to prove better growth kinetics of dental pulp stem cells as compared to other sources viz. bone marrow, umbilical cord and adipose tissue derived mesenchymal stem cells.

\subsubsection{PD time}

PD time studied at passage five has been reported in the Figure 1: PD time of MSCs across various stem cell sources. PD time was calculated using formula: $\mathrm{TD}=\mathrm{tp} \lg 2 /(\operatorname{lgNH}-\operatorname{lgNI})$. NI: the inoculums cell number; NH: the cell harvest number, and t: the time of the culture (in hours). Data is presented in hours.

As stated, dental pulp stem cells showed the lowest PD time followed by umbilical cord stem cells. This was one of the pivotal observation of the study suggesting dental pulp stem cell advantage over other sources.

\subsubsection{PD/cumulative $P D$}

PD and cumulative PD across different stem cell sources have been depicted in Figure 2: PD of MSCs across different stem cell sources and Figure 3: Cumulative PD of MSCs across various stem cell sources respectively. PD was determined by the formula:

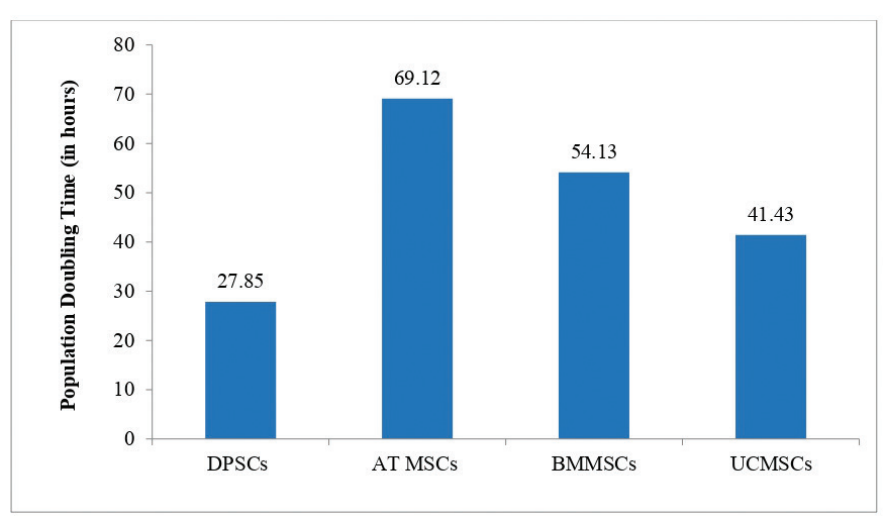

Figure 1: PD time of MSCs across various stem cell sources. Abbreviations: DPSCs = dental pulp stem cells; AT MSCs = Adipose tissue mesenchymal stem cells; BMMSCs = bone marrow mesenchymal stem cells; UCMSCs = umbilical cord mesenchymal stem cells.
$\mathrm{PD}=\mathrm{Log} \mathrm{NH}-\log \mathrm{NI} / \log 2 ;$ where NI: number of cells at seeding time and $\mathrm{NH}$ : number of cells at harvesting time. Cumulative Population Doubling (CPD) was determined by the formula: $\mathrm{CPD}=\mathrm{PD}$ of each passage $+\mathrm{PD}$ of previous passage [9].

Study results corroborated with PD time and maximum number of PD and cumulative PD were observed in descending order for dental pulp stem cells, umbilical cord mesenchymal stem cells, bone marrow mesenchymal stem cells and adipose tissue MSCs.

\subsubsection{Cell yield}

Cell yield obtained at end of passage five (P5) are reported in the Figure 4: Cell yield of MSCs across various stem cell sources. Highest cell yield was observed for dental pulp stem cells as compared to other sources.

This comparative study was conclusive on 'dental stem cell advantage' over other sources. Along with 'collection benefits', dental pulp stem cells were proved to be an optimal source of stem cells for producing conditioned medium.

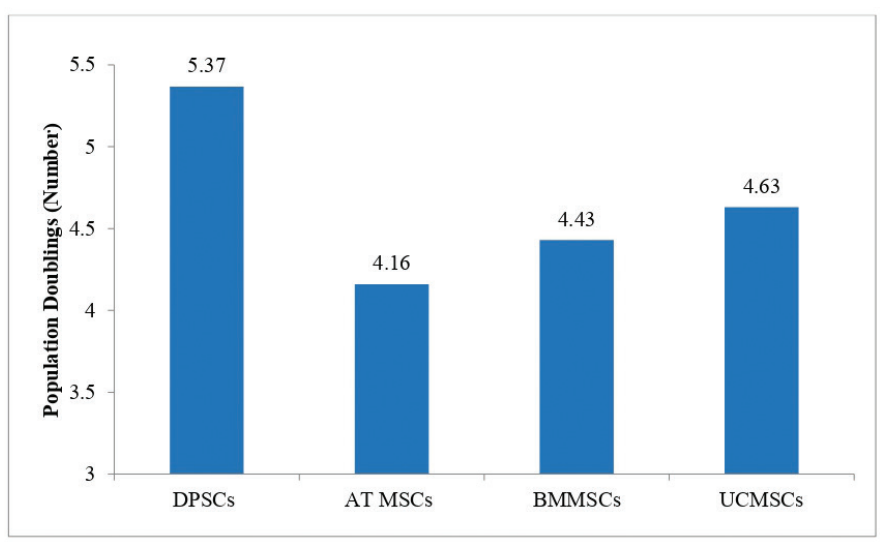

Figure 2: PD of MSCs across different stem cell sources. Abbreviations: DPSCs = dental pulp stem cells; AT MSCs = Adipose tissue mesenchymal stem cells; BMMSCs $=$ bone marrow mesenchymal stem cells; UCMSCs = umbilical cord mesenchymal stem cells.

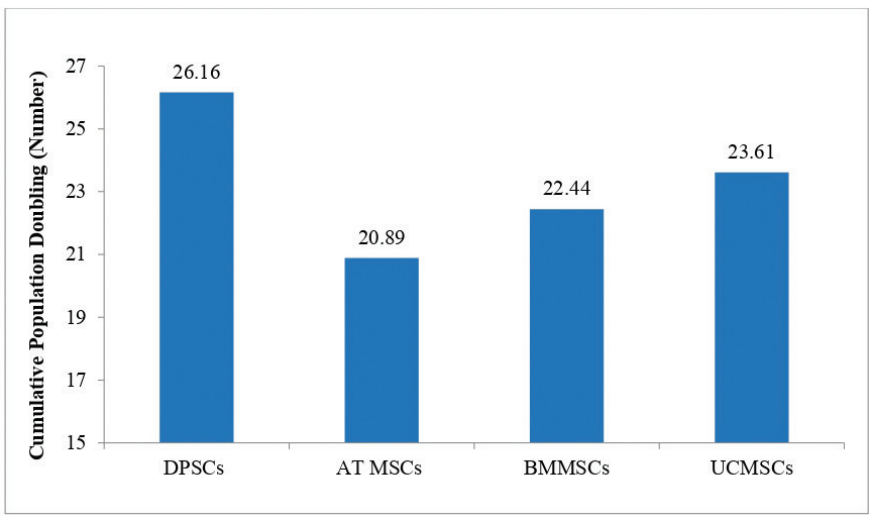

Figure 3: $\mathrm{CPD}$ of MSCs across various stem cell sources. Abbreviations: DPSCs = dental pulp stem cells; AT MSCs = Adipose tissue mesenchymal stem cells; $\mathrm{BMMSCs}=$ bone marrow mesenchymal stem cells; UCMSCs = umbilical cord mesenchymal stem cells. 


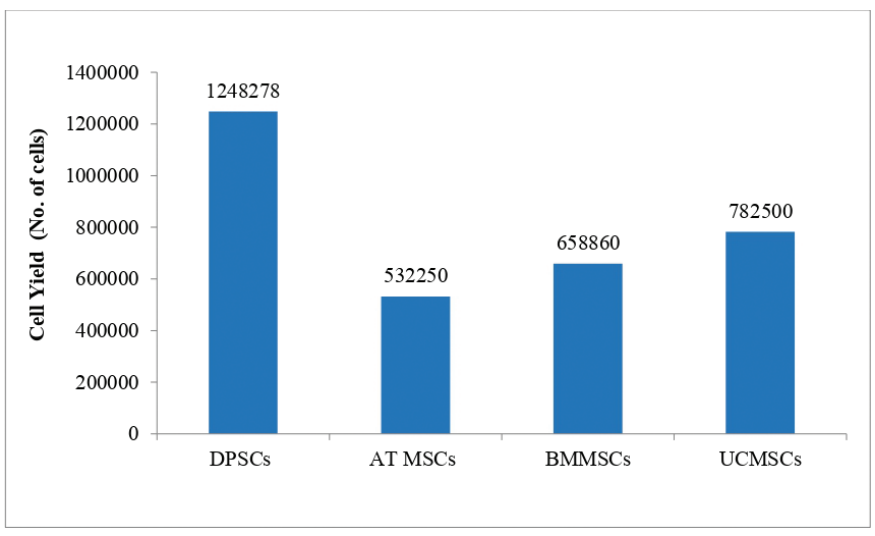

Figure 4: Cell yield of MSCs across various stem cell sources. Abbreviations: DPSCs $=$ dental pulp stem cells; AT MSCs $=$ Adipose tissue mesenchymal stem cells; BMMSCs $=$ bone marrow mesenchymal stem cells; UCMSCs = umbilical cord mesenchymal stem cells.

\subsection{Cell Viability of 'Pooled' Population of Dental Pulp Stem Cells}

Pooled biologics have been proved to be better than autologous biologics as per published studies [10]. It can be postulated that best cells persist along the doctrine of 'survival of fittest' phenomenon. Hence, for this research, stem cells sourced from Master Cell Bank were pooled and expanded to create Working Cell Bank. Significant improvement in product specification parameters reinforced the advantage of using pooled population as compared to individual samples stored in Master Cell Bank. The decreased standard deviation amongst the samples as shown in Figure 5 - Comparative Analysis - before and after pooling of stem cells, reinforce the point of reproducibility and thereby benefit of using pooled population of stem cells.

Improvement was observed in viability percentages and stem cell marker expression results. While viability percentages before pooling ranged from between $86 \%$ to $95 \%$, post pooling viability percentage of over $94 \%$ was recorded. Cells showed a positive expression of over $97 \%$ for CD90 and C105 and negative expression for CD31 (less than 1\%) post pooling. Spindle shaped morphology of dental pulp stem cells remained intact during the study.

Working Cell Bank of pooled population of dental pulp stem cells with superior viability percentage and expression with stem cell markers reinforced the reproducibility advantage assuring limited batch-to-batch variations during manufacture of conditioned medium on a commercial scale. Further, considering ease of availability of DPSCs, robustness of processing protocol, scope for large-scale expansion maintaining their stem cell properties, research exemplified possibility of creating a large working cell bank, an edge over other stem cell sources.

\subsection{Impact of Different Preconditioning Factors on Cytokine Secretion at Passage Level 5 (P5) Tested after 48 Hours}

\subsubsection{Comparative impact of different preconditioning factors when used in isolation}

Human or bovine sera are usually added to culture media to stimulate cell growth and proliferation as they provide basic

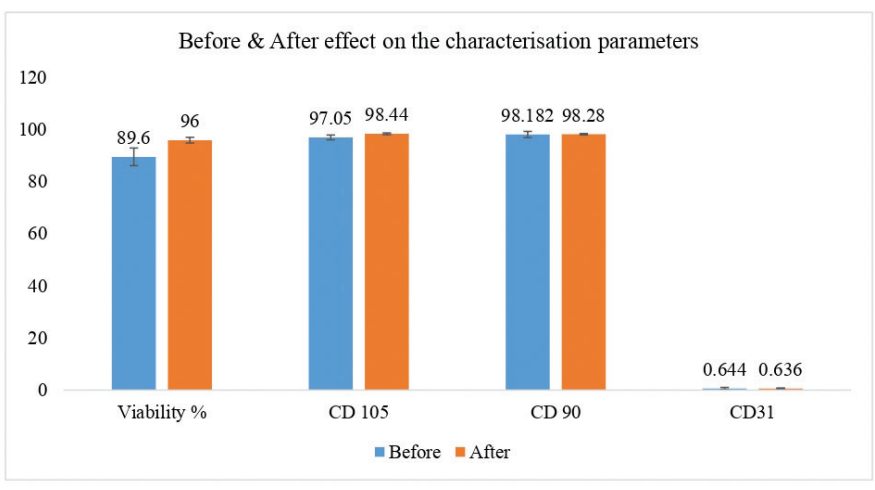

Figure 5: Comparative analysis — before and after pooling of stem cells.

nutrients, help in cell attachment, minimise mechanical damage and aid in $\mathrm{pH}$ maintenance. However, limited safety profile with high risk of contamination, donor variation hindering reproducibility have been deterrents to use in therapeutic applications. Better reproducibility and safety profile are promised by serum free media, however they are not considered at par to serum in promoting cell proliferation [11]. Since the intent of the study was to develop a conditioned medium for human use, reproducibility and safety profile were considered prime in making a choice of culture medium. Hence, serum free medium was chosen for producing conditioned medium.

Effect of various preconditioning factors on secretion of selective cytokines was compared against conditioned medium using serum free media as control. During this research, preconditioning factors encompassed chemical component like deferoxamine, growth factors and hormones such as Epidermal Growth Factor (EGF), Angiotensin I and ITS and vitamins like Niacinamide. Selection was done consciously to represent segments of chemicals, growth factors and vitamins.

Deferoxamine: Selection of this chemical component was based on earlier studies wherein preconditioning of MSCs culture medium with deferoxamine increased secretion of hypoxia inducible factor (HIF) - alpha maintaining characteristics of stemness and cell survival [12].

EGF: Recent studies have demonstrated short-term pretreatment with EGF induce high quantity of proteins and has been considered one of the key preconditioning regime to improve secretion of proteins in secretomes [13].

Niacinamide/Nicotinamide: This vitamin was known to play a pivotal role in Adenosine Triphosphate (ATP) production and thereby in cell proliferation.

Angiotensin I: Recent studies have indicated pretreatment of MSCs with Angiotensin II (Angiotensin I is a precursor to Angiotensin II) enhanced paracrine production of VEGF and angiogenesis [14].

ITS: ITS has been widely used to supplement culture medium to reduce amount of FBS. Since serum free medium was chosen for creation of Final Conditioned Medium, ITS had been identified as one of preconditioning factors.

Study results have been illustrated in Figure 6-Comparative Analysis-Cytokine secretions in isolation and combination. 


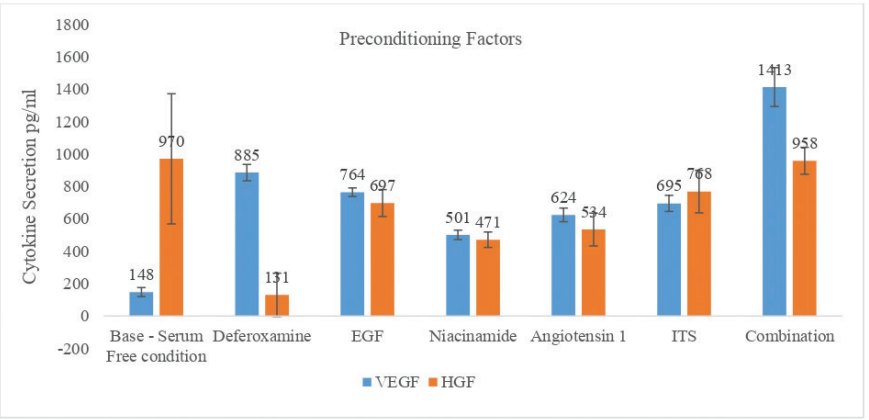

Figure 6: Comparative analysis - cytokine secretions in isolation and combination

Observations across preconditioning factors have been explained in detail below:

- Deferoxamine: there was a sixfold increase in VEGF secretion as compared to serum free media used as a control. However, use of deferoxamine decreased secretion of HGF. Recent data has shown that usage of iron chelator deferoxamine $(150 \mu \mathrm{M}$ or $400 \mu \mathrm{M})$ as preconditioning factor during development of adipose derived conditioned medium increased quantum of HIF which in turn increased secretions of cytokines like VEGF and angiopoetin 1 without impacting morphology and mesenchymal properties of cells [15]. Study results corroborated with published reports.

- Epidermal growth factor: Similar to deferoxamine, an upregulation in secretion of VEGF with EGF but the quantum increase was not as high as observed with deferoxamine. Downregulation for HGF was observed during research although the downregulation of HGF was not as significant as observed for deferoxamine.

- Niacinamide: Niacinamide is one of several vitamins essential for cell proliferation. It acts as a precursor for energy cofactors such as nicotinamide adenine dinucleotide, nicotinamide adenine dinucleotide phosphate and their reduced forms NADH and NADPH. These cofactors act as essential coenzymes in ATP production and as substrates for nuclear enzyme poly-ADPribose polymerase-1. It has been used as a major component in wide range of cell culture media including classical and serum free media. Representing vitamins amongst preconditioning factors, niacinamide was used to boost level of cytokines/growth factor. Similar to EGF, upregulation was observed in VEGF while downregulation was seen for HGF in comparison to serum free medium.

- Angiotensin I: Similar to published reports, this factor showed fourfold increase in VEGF secretion while downregulation of HGF was recorded in comparison to serum free medium.

- ITS: Insulin promotes glucose and amino acid uptake, lipogenesis, intracellular transport, and the synthesis of proteins and nucleic acids. Transferrin is an iron carrier and it may also help to reduce toxic levels of oxygen radicals and peroxide. Selenium, as sodium selenite, is a co-factor for glutathione peroxidase and other proteins, and is used as an anti-oxidant in media. As expected, ITS showed fivefold increase in VEGF secretion in comparison to serum free medium. However, downregulation was observed for HGF again.

\subsubsection{Comparative Impact of different preconditioning factors}

As depicted in Figure 6-Comparative Analysis-Cytokine secretions in isolation and combination, there was significant upregulation in secretion of VEGF and similar HGF levels when compared to readings obtained with serum free condition. VEGF secretion was almost 10 times of serum free condition. Enumerating the unique features of developed conditioned medium when preconditioning factors were used synergistically(1) Quantum of VEGF secretions was almost at par to serum containing medium. (2) VEGF was secreted in quantities needed for therapeutic effectiveness. (3) VEGF quantities in the developed conditioned medium were comparable to marketed products. Undoubtedly, with use of ultrafiltration techniques and established hypoxic preconditioning, VEGF secretion is likely to increase multifold further increasing the efficacy of conditioned medium. Additionally, HGF levels too were comparable to serum free medium conditioned medium when used in combination as shown in Figure 6. Here too, narrowing of standard deviation ranges in comparison to control stand evidence to the reduced variability in cytokine secretions and thereby reproducibility benefits. It has been a first time where multiple preconditioning factors were used to develop condition medium.

\section{CONCLUSION}

This exploratory study comprising comparative analysis of varied preconditioning factors, in isolation and combination, attempts to address two prime issues in current stem cell medium product development process. First, the study conclusively demonstrates the ability to increase/modify cytokine secretions in stem cell conditioned medium through right choice and right interplay of preconditioning factors, especially in cases of low concentrations of growth factors/cytokines. Possibility of selectively titrating varied preconditioning factors to control cytokine secretions has been conclusively proven in the study. This study is a good starting point to optimize culture conditions and selectively increase growth factors as demanded by the medical/research condition. This study is unique in its comparative analysis of a wide range of preconditioning factors, used both in isolation and combination in the same experimental design, thus eliminating all biases. Second, the study drives the possibility of the use of pooled population of HDPSCs for developing a commercially viable conditioned medium product. Better growth kinetics of dental stem cells help in building larger working stem cells banks, thereby aiding better scalability. One of the unique features of this study is the use of pooled population of stem cells, which opens up an additional avenue of consideration in the product development process. This investigation sets the foundation for future possibility of producing "customized" conditioned medium products aligned to clinical needs by controlling upregulation or downregulation of cytokines through an interplay of various physicochemical parameters. 


\section{CONFLICT OF INTEREST}

The authors declare that they do not have any conflicts of interest.

\section{FINANCIAL SUPPORT}

None.

\section{REFERENCES}

1. Drago D, Cossetti C, Iraci N, Gaude E, Musco G, Bachi A, Pluchino $\mathrm{S}$. The stem cell secretome and its role in brain repair. Biochimie 2013;95(12):2271-85.

2. Vizoso FJ, Eiro N, Cid S, Schneider J, Perez-Fernandez R. Mesenchymal stem cell secretome: toward cell-free therapeutic strategies in regenerative medicine. Int J Mol Sci 2017;18(9):1852.

3. Carriere A, Ebrahimian TG, Dehez S, Auge N, Joffre C, Andre M, et al. Preconditioning by mitochondrial reactive oxygen species improves the proangiogenic potential of adipose-derived cells-based therapy. Arterioscl Throm Vas Biol 2009;29(7):1093-9.

4. Liu L, Gao J, Yuan Y, Chang Q, Liao Y, Lu F. Hypoxia preconditioned human adipose derived mesenchymal stem cells enhance angiogenic potential via secretion of increased VEGF and bFGF. Cell Biol Int 2013;37(6):551-60.

5. Chacko SM, Ahmed S, Selvendiran K, Kuppusamy ML, Khan M, Kuppusamy P. Hypoxic preconditioning induces the expression of prosurvival and proangiogenic markers in mesenchymal stem cells. Am J of Physiol Cell Physiol 2010;299(6):C1562-70.

6. Miura M, Gronthos S, Zhao M, Lu B, Fisher LW, Robey PG, et al. SHED: stem cells from human exfoliated deciduous teeth. Proc Natl Acad Sci USA 2003;100(10):5807-12.

7. Nakashima M, Iohara K, Murakami M. Dental pulp stem cells and regeneration. Endod Top 2013;28:38-50.

8. Hass R, Kasper C, Böhm S, Jacobs R. Different populations and sources of human mesenchymal stem cells (MSC): a comparison of adult and neonatal tissue-derived MSC. Cell Commun Signal 2011;9:12.
9. Bronckaers A, Hilkens P, Fanton Y, Struys T, Gervois P. Angiogenic properties of human dental pulp stem cells. PLoS One 2013;8(8):e71104.

10. Munirah S, Ruszymah BH, Samsudin OC, Badrul AH, Azmi B, Aminuddin BS. Autologous versus pooled human serum for articular chondrocyte growth. J Orthop Surg (Hong Kong) 2008;16(2):220-9.

11. Froud SJ. The development, benefits and disadvantages of serum-free media. Dev Biol Stand 1999;99:157-66.

12. Hyun JL, Young HJ, Gee EC, Jun SK, Chang WC, Ho JH. Role of HIF1 $\alpha$ Regulatory factors in stem cells. Int J Stem Cells 2019;12(1):8-20.

13. Baer PC, Overath JM, Urbschat A, Schubert R, Geiger H. Preconditioning of human adipose-derived stromal/stem cells: evaluation of short-term preincubation regimens to enhance their regenerative potential. J Stem Cell Res Ther 2016;6:3.

14. Liu C, Fan Y, Zhou L, Zhu HY, Song YC, Hu L, et al. Pretreatment of mesenchymal stem cells with angiotensin II enhances paracrine effects, angiogenesis, gap junction formation and therapeutic efficacy for myocardial infarction. Int J Cardiol 2015;188:22-32.

15. Oses C, Olivares B, Ezquer M, Acosta C, Bosch P, Donoso M, et al. Preconditioning of adipose tissue-derived mesenchymal stem cells with deferoxamine increases the production of pro-angiogenic, neuroprotective and anti-inflammatory factors: potential application in the treatment of diabetic neuropathy. PLoS One 2017;12(5):e0178011.

\section{How to cite this article:}

Mokashi R, Dasgupta D. Assessment of impact of varied preconditioning factors, used in isolation and combination, for enhancement of cytokine secretions in stem cell conditioned medium. J Appl Biol Biotech 2020;8(03):051-056. DOI: 10.7324/JABB.2020.803010 